\title{
AN INEQUALITY FOR CERTAIN PENCILS OF PLANE CURVES
}

\section{A. GUTWIRTH ${ }^{1}$}

1. Introduction. In this paper we use a method similar to one used by Engel in [1] in order to get an inequality relating the degree of the generic curve of a rational pencil of plane curves with the order of its divisor of singularities at a base point of the pencil satisfying some special conditions. In [1] Engel uses a particular case of this relation to give a new proof of the following theorem of Jung:

Consider the affine plane over the field $k$ of complex numbers. Then any entire Cremona transformation is a product of linear transformations and transformations of the following type:

$$
\begin{aligned}
& x^{\prime}=x, \\
& y^{\prime}=y+c x^{n}
\end{aligned}
$$

where $c \in k$ and $n$ is a positive integer.

For the convenience of the reader we sketch how one can obtain a simple proof of the preceding result using our Corollary A in \$7.

Our Lemma B is an abstraction and generalization of a lemma of Jung [2], and is proved in more generality than needed here for further reference.

2. Definitions. Let $k$ be an algebraically closed field of characteristic zero, $m$ and $(m-\alpha)$ two integers, $d$ their g.c.d., so that $m=a d$ and $(m-\alpha)=b d$, where $a$ and $b$ are relatively prime.

Consider in the projective plane over $k$ an irreducible curve of order $m$ having a singular point $P$ such that:

(1) $P$ is the center of $n$ places, all having the same tangent.

(2) For each $i=1, \cdots, n$ there exists an integer $t_{i}$ such that a linear form has order $a t_{i}$ or $b t_{i}$ at the $i$ th place.

(3) $\sum t_{i}=d$ (so that $\sum a t_{i}=m, \sum b t_{i}=m-\alpha$ ). Such a point will be called an $(m, m-\alpha)$-point. Such a curve will be called an $(m, m-\alpha)$ curve. A pencil of curves, depending linearly on a parameter $\lambda$, whose generic member has an $(m, m-\alpha)$-point that is independent of $\lambda$, and has its tangent independent of $\lambda$ will be called an $(m, m-\alpha, \lambda)$ pencil.

Received by the editors January 19, 1960, and, in revised form, March 19, 1960, and August 1, 1960.

1 This paper was sponsored by the National Science Foundation under Contract No. NSF G 9657. 
As the property of being an $(m, m-\alpha)$-curve is a local one we will use affine coordinates.

3. Lemma A. Given an $(m, m-\alpha, \lambda)$-pencil, a system of affine coordinates [in the projective plane] can be so chosen that the corresponding equation $F(x, y, \lambda)=0$ admits the following factorization:

$$
F(x, y, \lambda)=h \prod_{\epsilon \delta_{\epsilon}}\left(y-y_{\epsilon \delta_{\epsilon}}\right)
$$

where

$h$ is a unit in $\mathrm{Cl} k(\lambda)[y]\left[\left[x^{1 / b t}\right]\right]$,

the $y_{\epsilon \delta_{\epsilon}}$ are nonunits in $\mathrm{Cl} k(\lambda)\left[\left[x^{1 / b t}\right]\right]$,

e ranges from 1 to $n$,

$\delta_{\epsilon}$ ranges from 1 to $b t_{\epsilon}$,

$t$ is the l.c.m. of the $t_{i}$ 's,

$\mathrm{Cl}$ denotes algebraic closure and the double bracket formal powerseries ring.

Proof. It suffices to take as origin the $(m, m-\alpha)$-point, and $y=0$ as the tangent to the $n$ corresponding places. The desired factorization is then obtained in the standard way, taking into account the existence of Puiseux-expansions at the origin and the form of the local Galois-group at that point.

Note that for the $i$ th place there will be $b t_{i}$ factors in this factorization, each of them of the form:

$$
y-D_{0}\left(\theta^{\eta} x^{1 / b t_{i}}\right)^{a t_{i}}-\sum_{v=1}^{\infty} D_{v}\left(\theta^{\eta} x^{1 / b t_{i}}\right)^{a t_{i}+v}
$$

where $\theta$ is a primitive $b t_{i}$ th root of unity and $D_{i} \in \mathrm{Cl} k(\lambda)$.

4. LemmA B. Let $k$ be any field, $y x_{1} \cdots x_{n}$ and $\lambda$ indeterminates. Suppose we have an identity

$$
g\left(y x_{1} \cdots x_{n}\right)+\lambda f\left(y x \cdots x_{n}\right)=h \prod_{\epsilon=1}^{m}\left(y-y_{\epsilon}\right)
$$

where

$g$ and $f$ are elements of $k\left[\left[x_{1} \cdots x_{n}\right]\right][y]$,

the $y_{i}$ are nonunits in $\mathrm{Cl} k(\lambda)\left[\left[x_{1} \cdots x_{n}\right]\right]$,

$h$ is a unit in $\mathrm{Cl} k(\lambda)\left[\left[y x_{1} \cdots x_{n}\right]\right]$.

Order the terms of the power-series $y_{\mathrm{c}}$ lexicographically in the $x_{1} \cdots x_{m}$. In some of the $y_{\epsilon}$ certain coefficients may depend on $\lambda$. Suppose that the first such coefficient in every $y_{\epsilon}$ is separable over $k(\lambda)$. Then if two at least of the $y_{\mathrm{c}}$ coincide in their first $j$ terms the coefficients of these terms do not depend on $\lambda$. 
Proof. Suppose that in $\sigma \geqq 2$ of the $m$ power series $y_{\epsilon}$, say for $\epsilon=1, \cdots, \sigma$, the first term whose coefficient depends on $\lambda$ is $a(\lambda) x_{1}^{\mu_{1}} \cdots x_{n}^{\mu_{n}}$, of total degree $r=\mu_{1}+\cdots+\mu_{n}$, and suppose that these series coincide up to this last term at least.

Define $y^{*}$ as a polynomial in the $x_{1} \cdots x_{n}$ such that

(1) $y^{*}=y_{1}$ (modulo terms of degree $r$ ),

(2) $y^{*}$ has as terms of degree $r$ the same terms as $y_{1}$ up to $a(\lambda) x_{1}^{\mu_{1}} \cdots x_{n}^{\mu_{n}}$, and instead of this term has the term $t x_{1} \mu_{1} \cdots x_{n}^{\mu_{n}}$ where $t$ is an indeterminate and has no higher terms. Note that $y^{*}$ is linear in $t$. In $\mathrm{Cl} k(\lambda)\left[\left[y x_{1} \cdots x_{n} t\right]\right]$ we consider for (4.1) the specialization $y \rightarrow y^{*}$. We then get

$$
g\left(y^{*} x_{1} \cdots x_{n}\right)+\lambda f\left(y^{*} x_{1} \cdots x_{n}\right)=h^{*} \prod_{\epsilon=1}^{m}\left(y^{*}-y_{\epsilon}\right)
$$

where $g\left(y^{*} x_{1} \cdots x_{n}\right)$ and $f\left(y^{*} x_{1} \cdots x_{n}\right)$ are polynomials ${ }^{*}$ in $t$. Put

$$
\begin{aligned}
& g\left(y^{*} x_{1} \cdots x_{n}\right)=g^{*}\left(t x_{1} \cdots x_{n}\right), \\
& f\left(y^{*} x_{1} \cdots x_{n}\right)=f^{*}\left(t x_{1} \cdots x_{n}\right) .
\end{aligned}
$$

Obviously $h^{*}$ remains a unit in $\mathrm{Cl} k(\lambda)\left[\left[y x_{1} \cdots x_{n} t\right]\right]$.

In $\left(y^{*}-y_{\epsilon}\right)$ the first term of every factor $\left(y^{*}-y_{\epsilon}\right)$ will have as coefficient

$$
t-a(\lambda) \quad \text { if } \epsilon=1,2, \cdots, \sigma,
$$

and for any other $\epsilon$ it may be $t-c_{\epsilon}(\lambda)$ or an expression $d_{\epsilon}(\lambda)$ independent of $t$, or $t$. By hypothesis $a(\lambda), c_{\epsilon}(\lambda), d_{\epsilon}(\lambda)$ are separable over $k(\lambda)$. The first of the lowest degree terms in the right hand side of (4.2) will be obtained by multiplying the first terms in every factorthis gives a term of the form:

$$
[t-a(\lambda)]^{\sigma} \phi(t, \lambda) x_{1}^{\xi_{1}} \cdots x_{n}^{\xi_{n}}
$$

where $\phi$ is a polynomial in $t$ whose coefficients are separable over $k(\lambda)$.

Therefore

$g^{*}\left(t x_{1} \cdots x_{n}\right)+\lambda f^{*}\left(t x_{1} \cdots x_{n}\right) \equiv[t-a(\lambda)]^{\sigma} \phi x_{1}^{\xi_{1}} \cdots x_{n}^{\xi_{n}}$

+different terms of same total degree + terms of higher degree.

Let $A(t) x_{1}^{\xi_{1}} \cdots x_{n}^{\xi_{n}}$ and $B(t) x_{1}^{\xi_{1}} \cdots x_{n}^{\xi_{n}}$ be the first of the lowestdegree terms in $g^{*}\left(t x_{1} \cdots x_{n}\right)$ and $f^{*}\left(t x_{1} \cdots x_{n}\right)$ respectively- $A$ and $B$ are polynomials in $t$.

Then 


$$
A(t)+\lambda B(t)=[t-a(\lambda)]^{\sigma} \phi(t, \lambda) .
$$

Differentiating with respect to $\lambda$ we get

$$
B(t)=[t-a(\lambda)]^{\sigma-1} \psi(t, \lambda) \quad(\psi \text { polynomial in } t)
$$

and $B(t)$ has $t=a(\lambda)$ as a root-a contradiction, since $B(t)$ is independent of $\lambda$.

5. MaIn THEOREM. Consider an $(m, m-\alpha, \lambda)$-pencil in whose generic member the order of the divisor of singularities at its $(m, m-\alpha)$-point is equal to $c$. Then the following inequality holds:

$$
m^{2}+\alpha+n \geqq \alpha a+2 m+c .
$$

Proof. By Lemma A we can choose a system of coordinates such that the corresponding equation of the $(m, m-\alpha, \lambda)$-pencil, $F(x, y, \lambda)$ $=0$, admits the factorization:

$$
F=h \prod_{\epsilon, \delta_{\epsilon}}\left(y-y_{\epsilon \delta_{\epsilon}}\right) .
$$

It is well known (see [3]) that the order of the divisor of singularities at $P$ for a curve $F=0$ is:

$$
c=-\sum_{i=1}^{n} V_{P_{i}}\left[\frac{d x}{F_{y}}\right]
$$

the sum ranging over the $n$ places of $F=0$ at $P$. For the $(m, m-\alpha)$ curves we consider we then have:

$$
\sum V_{P_{i}}\left[F_{y}\right]=c+\sum V_{P_{i}}[d x]=c+m-\alpha-n .
$$

We will now compute an upper bound for $V_{P_{i}}\left[F_{y}\right]$ using (5.1).

We have

$$
\sum V_{P_{i}}\left[F_{\nu}\right]=\sum V_{P_{i}}\left[\frac{\partial}{\partial y} h \prod_{\epsilon, \delta_{\epsilon}}\left(y-y_{\epsilon \delta_{\epsilon}}\right)\right] .
$$

At $P_{i}$ we identify $y$ with one of the roots $y_{i 1}, \cdots, y_{i b t_{i}}$, say with $y_{i 1}$. Then

$$
\sum V_{P_{i}}\left[F_{y}\right]=\sum_{i} V_{P_{i}} \prod_{\epsilon \delta_{\epsilon \in i 1}}\left(y-y_{\epsilon \delta_{e}}\right) .
$$

We compute $V_{P_{i}} \prod_{\delta_{i} \neq 1}\left(y-y_{i \delta_{i}}\right)$ first. Only for $t_{i}$ of the $\delta_{i}$ will the first coefficient of $y_{i i_{i}}$ be equal to the first coefficient of $y_{i 1}$, and in that case, by Lemma B, the two expansions $y_{i b_{i}}$ and $y_{i 1}$ coincide at most up to the first term whose coefficient depends on $\lambda$. Denote by 
$\rho(\lambda) x\left(a t_{i}+\beta_{i}\right) / b t_{i}$ the first term in $y_{i 1}$ whose coefficient depends on $\lambda$. Then

$$
V_{P_{i}} \prod_{\delta_{i} \neq 1}\left(y-y_{i i_{i}}\right) \leqq\left(t_{i}-1\right)\left(a t_{i}+\beta_{i}\right)+\left(b t_{i}-t_{i}\right) a t_{i} .
$$

For the remaining part, $V_{P_{i}} \Pi_{j \neq 1}\left(y-y_{j \delta_{j}}\right)$ a similar argument gives

$$
V_{P_{i}} \prod_{j \neq 1}\left(y-y_{j b_{j}}\right) \leqq t_{j}\left(a t_{i}+\beta_{i}\right)+\left(b t_{j}-t_{j}\right) a t_{i} .
$$

All in all,

$$
\begin{aligned}
V_{P_{i}}\left[\frac{\partial}{\partial y} h \Pi\left(y-y_{e \delta_{e}}\right)\right] & \\
& \leqq\left(a t_{i}+\beta_{i}\right)\left(\sum t_{j}-1\right)+a t_{i}\left[\sum_{j}\left(b t_{j}-t_{j}\right)\right] .
\end{aligned}
$$

Remembering that $\sum t_{i}=d$ we get

$$
V_{P_{i}}\left[F_{y}\right] \leqq a b d t_{i}-a t_{i}+(d-1) \beta_{i} .
$$

Taking the sum over all the places centered at $P$ we have:

$$
\sum_{i} V_{P_{i}}\left[F_{y}\right] \leqq a b d^{2}+(d-1) \sum \beta_{i}-a d .
$$

To evaluate $\sum \beta_{i}$ we consider two independent generic members of the pencil, with parameters $\lambda, \mu$ and the expression:

$$
V_{P_{i}}\left(y_{i \delta_{i}}(\mu)-y_{\epsilon \delta_{\epsilon}}(\lambda)\right) \text { with } i \delta_{i} \neq \epsilon \delta_{\epsilon},
$$

the $P_{i}$ 's being taken on the $\lambda$-curve.

Using Lemma B we get in this case

$$
V_{P_{i}}\left(y_{i \delta_{i}}(\mu)-y_{\epsilon \delta_{\epsilon}}(\lambda)\right)=V_{P_{i}}\left(y_{i \delta_{i}}(\lambda)-y_{\epsilon \delta_{\epsilon}}(\lambda)\right) .
$$

Therefore

$$
\sum_{i} V_{P_{i}} \prod_{\epsilon \delta_{\epsilon \neq i 1}}\left(y_{i 1}(\mu)-y_{\epsilon \delta_{\epsilon}}(\lambda)\right)=\sum V_{P_{i}}\left[F_{y}\right] .
$$

But the intersection-multiplicity of two generic members of the pencil at $P$ is

$$
\begin{aligned}
& \sum_{i} V_{P_{i}} \prod\left(y_{i 1}(\mu)-y_{\epsilon \delta_{\epsilon}}(\lambda)\right) \\
& \quad=\sum_{i} V_{P_{i}} \prod_{\epsilon \delta_{\epsilon \neq i 1}}\left(y_{i 1}(\mu)-y_{\epsilon \delta_{\epsilon}}(\lambda)\right)+\sum_{i} V_{P_{i}}\left(y_{i 1}(\mu)-y_{i 1}(\lambda)\right) .
\end{aligned}
$$

Moreover $\sum_{i} V_{P_{i}}\left(y_{i 1}(\mu)-y_{i 1}(\lambda)\right)=\sum_{i}\left(a t_{i}+\beta_{i}\right)=m+\sum \beta_{i}$. This 
multiplicity is $\leqq m^{2}$, so that $\sum V_{P_{i}}\left[F_{y}\right]+m+\sum \beta_{i} \leqq m^{2}$. By (5.2) we have:

$$
\sum \beta_{i} \leqq m^{2}-2 m-c+\alpha+n .
$$

From (5.2) and (5.6) follows:

$$
c+m-\alpha-n \leqq a b d^{2}-a d+(d-1) \sum \beta_{i} .
$$

A fortiori,

$$
c+m-\alpha-n \leqq a b d^{2}-a d+(d-1)\left(m^{2}-2 m-c+\alpha+n\right)
$$

or finally

$$
d m^{2}+\alpha d+n d \geqq \alpha m+2 d m+c d .
$$

6. Proposition. Let the generic curve of an $(m, m-\alpha, \lambda)$-pencil have as order of its divisor of singularities at its $(m, m-\alpha)$-point the number $c$, with $c>(m-1)(m-2)-2$. Then $\alpha$ divides $m$.

Applying the main theorem,

$$
m^{2}+\alpha+n>\alpha a+2 m+(m-1)(m-2)-2=a \alpha+m^{2}-m,
$$

or

$$
m+n>\alpha(a-1) .
$$

Since $m=a d$ and $n \leqq d$ we have a fortiori

$$
a+1>\frac{\alpha}{d}(a-1) .
$$

As both $a>1$ and $d \mid \alpha$ this can only hold for $\alpha=d$.

COROLlaRy A. If the generic member of an $(m, m-\alpha, \lambda)$-pencil is a rational curve, and has no other singular point than its $(m-\alpha)$-point, then $\alpha$ divides $m$.

Proof. For the order of the divisor of singularities is $(m-1)(m-2)$ minus twice the genus.

7. JUNG'S THEOREM. Consider the affine plane over an algebraically closed field $k$ of characteristic 0 . Then any automorphism of this plane is a product of linear transformations and transformations of the following type:

$$
\begin{aligned}
& x^{\prime}=x, \\
& y^{\prime}=y+c x^{n},
\end{aligned}
$$

where $c \in k$ and $n$ is a positive integer. 
Proof. Let $\sigma$ be an automorphism of the plane given by

$$
\begin{aligned}
& x^{\prime}=f(x, y), \\
& y^{\prime}=y(x, y),
\end{aligned}
$$

where $f$ and $g$ are polynomials, elements of $k[x, y]$ of degree $n$ and $m$ respectively, $n \geqq m$. For $n=m=1$ there is nothing to prove. So we suppose $n \geqq 2$. As $\sigma$ is everywhere biregular in the affine plane the jacobian of $f$ and $g$ is constant. It then follows that the highest degree terms of $f$ and $g$, having jacobian zero, are, up to a constant factor, powers of a common polynomial $h$. In the projective plane $\sigma$ defines a birational transformation which is well defined for a generic point of infinity. The same is true for $\sigma^{-1}$, so that to the point at infinity on a generic line of the affine plane there corresponds under $\sigma^{-1}$ only one point at infinity on the corresponding curve. It follows that the polynomial $h$ is a power of a linear form.

By a linear transformation (7.1) becomes:

$$
\begin{aligned}
& x^{\prime}=x^{n}+f_{1}(x, y), \\
& y^{\prime}=x^{m}+g_{1}(x, y)
\end{aligned}
$$

where $n \geqq m, n \geqq 2$ and the degrees of $f_{1}$ and $g_{1}$ are respectively $\leqq n-1$, $\leqq m-1$. After factoring out a linear transformation if necessary we may assume $n>m$.

To the pencil $y=\lambda, \lambda$ an indeterminate, there corrresponds under $\sigma$ a pencil of rational curves. Consider their parametric representation, obtained from (7.2) by putting $y=\lambda$. It is readily seen that a generic member of this pencil has a unique point at infinity, $P$, independent of $\lambda$, center of only one place, with the line at infinity as tangent. Moreover a line through $P$ intersects the curve in either $n$ or $(n-m)$ points at $P$. Obviously a generic curve of the pencil has no singularities in the affine plane. Such a pencil is a particular case of a $(n, n-m, \lambda)$-pencil, and by Corollary A we get $m \mid n$.

Then $\sigma=w \circ \mu$, where $w$ is the transformation

$$
\begin{aligned}
& x^{\prime}=x^{\prime \prime}+y^{\prime \prime n / m}, \\
& y^{\prime}=y^{\prime \prime},
\end{aligned}
$$

and $\mu$ is of the type

$$
\begin{aligned}
& x^{\prime \prime}=p(x, y), \\
& y^{\prime \prime}=x^{m}+g_{1}(x, y),
\end{aligned}
$$

with $p$ a polynomial of degree less than $n$. This proves the theorem. 


\section{REFERENCES}

1. W. Engel, Ganze Cremona-Transformationen von Primzahlgrad in der Ebene, Math. Ann. vol. 136 (1958) pp. 319-325.

2. H. W. E. Jung, Einführung in die Theorie der algebraischen Functionen zweier Veränderlicher, Berlin, Akademie Verlag, 1951, p. 61.

3. P. Samuel, Singularitês des variêtés algébriques, Bull. Soc. Math. France vol. 79 (1951) pp. 121-129.

University of California, Berkeley

TEChNion, HaIFA, IsRaEL

\section{ON PROJECTIVE MODULES OVER SEMI-HEREDITARY RINGS}

\section{FELIX ALBRECHT}

This note contains a proof of the following

THEOREM. Each projective module $P$ over a (one-sided) semi-hereditary ring $\Lambda$ is a direct sum of modules, each of which is isomorphic with a finitely generated ideal of $\Lambda$.

This theorem, already known for finitely generated projective modules [1, I, Proposition 6.1], has been recently proved for arbitrary projective modules over commutative semi-hereditary rings by $\mathrm{I}$. Kaplansky [2], who raised the problem of extending it to the noncommutative case.

We recall two results due to Kaplansky:

Any projective module (over an arbitrary ring) is a direct sum of countably generated modules [2, Theorem 1 ].

If any direct summand $N$ of a countably generated module $M$ is such that each element of $N$ is contained in a finitely generated direct summand, then $M$ is a direct sum of finitely generated modules [2, Lemma 1].

According to these results, it is sufficient to prove the following proposition:

Each element of the module $P$ is contained in a finitely generated direct summand of $P$.

Let $F=P \oplus Q$ be a free module and $x$ be an arbitrary element of $P$. Let $x=\lambda_{1} x_{1}+\cdots+\lambda_{n} x_{n}$ be a representation of the element $x$ in some base for the free module $F$ and let $G$ denote the free submodule

Received by the editors June 28, 1960. 